\title{
Primary mucinous adenocarcinoma developing in an ileostomy stoma
}

\author{
P J SMART, S SASTRY, AND S WELLS \\ From the Departments of Histopathology and Surgery, Bolton General Hospital, Farnworth, Bolton, Lancs
}

SUMMARY We report a mucinous adenocarcinoma arising in an ileostomy stoma of a 60 year old woman after a subtotal colectomy done 28 years previously for ulcerative colitis. A study of the histochemical profiles of the mucin within the mucosa of the ileostomy and the carcinoma was undertaken. Previously documented cases are reviewed.

\section{Case history}

Mrs M S a 60 year old woman was seen at home on 11 December 1987 by the stoma nurse because of problems with her ileostomy. In February 1960 she underwent a subtotal colectomy and terminal ileostomy for ulcerative colitis, leaving the rectal stump in situ. She gave a four month history of a wart like lesion on her ileostomy associated with mucus discharge, bleeding and increased frequency of ileostomy function.

Examination showed a $4 \times 3 \times 2 \mathrm{~cm}$ polypoid tumour adjacent to the ileostomy (Fig. 1). Biopsies were taken and the patient admitted for excision of the tumour.

Under anaesthesia, the lesion was seen to be arising from the junctional area of the ileal mucosa and skin. On the basis of biopsy histology the lesion was locally excised using a crescentic incision, excising part of the junctional zone. The wound healed well postoperatively and further biopsies were later obtained from the edges of the ileostomy away from the area of the previous operation.

Mrs M S has no symptoms from the remaining rectal stump and the anus is fibrosed. At the time of writing, three months postoperatively, she is well, the ileostomy is functioning normally, and there is no evidence of recurrence of the tumour.

Address for correspondence: Dr S Wells, Department of Histopathology, Bolton General Hospital, Minerva Road, Farnworth, Bolton BL4 0JR.

Received for publication 1 June 1988.
PATHOLOGICAL FINDINGS

The initial biopsy consisted of two irregular pieces of pale tissue, the largest $0.6 \mathrm{~cm}$ in diameter. Histologically this contained papillae and glandular elements showing marked epithelial atypia, chronic inflammatory cell infiltration, and ulceration. These were

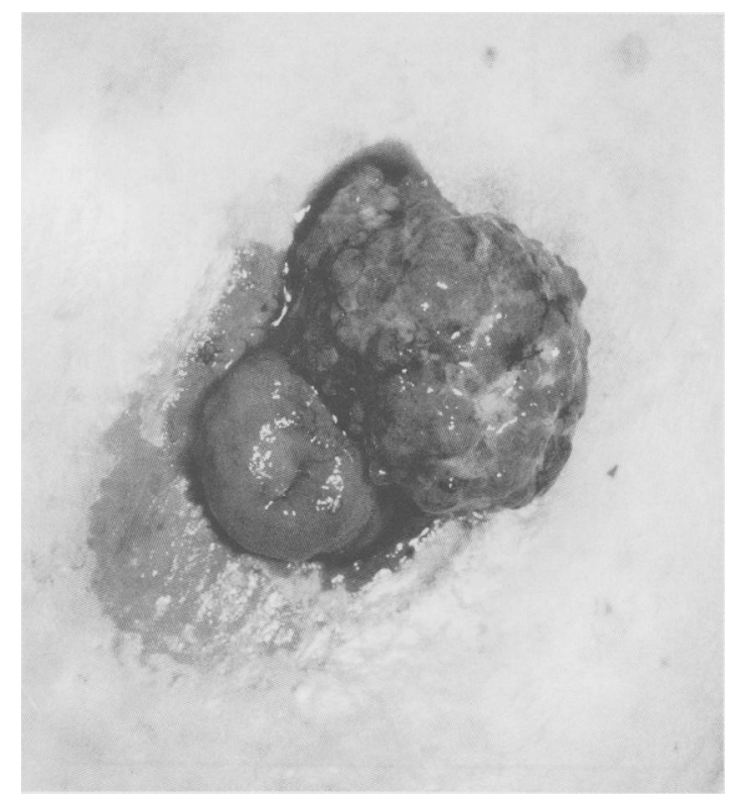

Fig. 1 Ileostomy stoma with polypoid tumour arising at its edge. 
interpreted as representing origin from a tubulovillous adenoma. Although invasive neoplasia could not be seen there was a possibility of invasive neoplasia in the remaining tumour.

The resected specimen consisted of an ellipse of skin $6 \times 2.2 \mathrm{~cm}$ with $1.2 \mathrm{~cm}$ of underlying fat. Situated on the surface was an irregular, multilobated tumour mass $3.2 \times 2.4 \times 1.8 \mathrm{~cm}$ apparently arising from the edge of the ileostomy stoma. The cut surface was multicystic and contained mucoid material (Fig. 2). Histologically the superficial part of the tumour had a pattern similar to that of a tubulovillous adenoma with marked epithelial dysplasia (Fig. 3) but, within the tumour base, invasive adenocarcinoma was readily identified. The invasive component of this lesion had a distinctive mucoid appearance with neoplastic cysts containing mucus (Fig. 4). All the invasive component was of this type and it was classified as a primary mucoid adenocarcinoma. The mucosa at the junction of the tumour and the skin

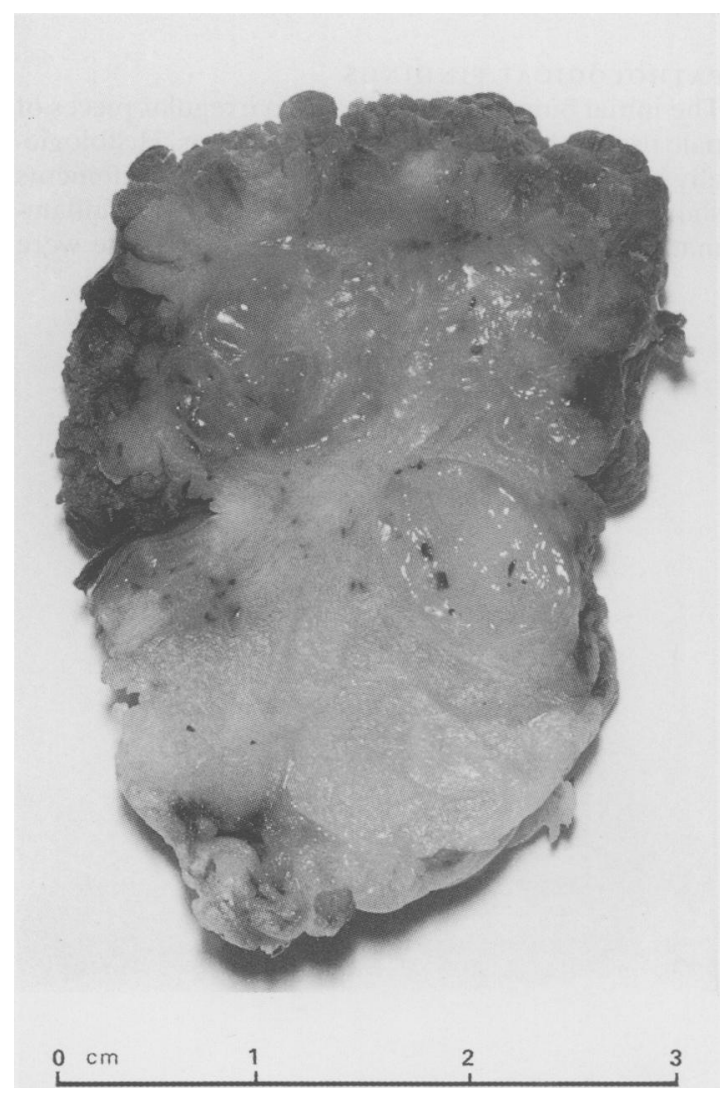

Fig. 2 Cut surface of the excised tumour illustrating the polypoidal appearance, skin tumour junction and mucin lakes in the deeper parts of the tumour.
(Fig. 5) showed loss of villous architecture and marked dysplasia, although some Paneth cells were readily identified.

The postoperative biopsies from the remaining stoma consisted of two pieces of pale tissue, the largest $0.4 \mathrm{~cm}$ across. Histologically (Fig. 6) this showed intestinal mucosa with muscularis mucosa. The mucosa was flattened, the glands contained Paneth cells at their bases and goblet cells were plentiful. There was no evidence of dysplasia or neoplasia in these specimens. The architecture within this biopsy was reminiscent of colonic mucosa and the appearances therefore suggested incomplete large intestinal metaplasia.

Pathological material from the previous colectomy 28 years previously was unfortunately not available for review but it was reported as ulcerative colitis and no tumour was found.

In order to determine whether this tumour had arisen in small bowel mucosa showing large bowel metaplasia, mucin studies were done to identify sulpho and sialo acidic mucins and neutral mucin using Spicer's High Iron Diamine Alcian Blue (HIDAB) method ${ }^{\prime}$ and periodic acid Schiffs reagent. To further characterise the sialo mucins into those that contained o-acyl groups, the periodate-borohydride, potassium hydroxide, PAS (PB/KOH/PAS) technique was used $\mathrm{d}^{2}$ on the resected specimen and the postoperative biopsy.

\section{Results}

These are summarised in the Table where the expected staining reactions of normal large and small intestine are included. ${ }^{3}$

\section{RESECTED SPECIMEN}

The epithelium within the tumour, the adjacent mucosa and the invasive mucoid areas all stained with Alcian Blue and PAS, indicating acid and neutral

Table Staining reactions

\begin{tabular}{|c|c|c|c|c|}
\hline & Neutral & Sialo & Sulpho & o-Acylated \\
\hline Normal ileum & + & ++ & - & - \\
\hline Normal proximal colon & ++ & $\begin{array}{l}++ \\
\text { lower } 1 / 3\end{array}$ & $\begin{array}{l}++ \\
\text { upper } 2 / 3\end{array}$ & ++ \\
\hline Normal distal colon & $\begin{array}{l}+ \\
\text { upper } \\
\text { crypt }\end{array}$ & $\begin{array}{l}++ \\
\text { upper 1/2 }\end{array}$ & $\begin{array}{l}++ \\
\text { lower } 1 / 2\end{array}$ & +++ \\
\hline \multicolumn{5}{|l|}{ Resected specimen } \\
\hline $\begin{array}{l}\text { Mucosa adjacent to } \\
\text { tumour }\end{array}$ & focal + & ++ & ++ & - \\
\hline Surface of tumour & + & - & ++ & - \\
\hline Mucoid areas of tumour & ++ & + & ++ & focal + \\
\hline Postoperative biopsy & ++ & + & $+t$ & focal + \\
\hline
\end{tabular}




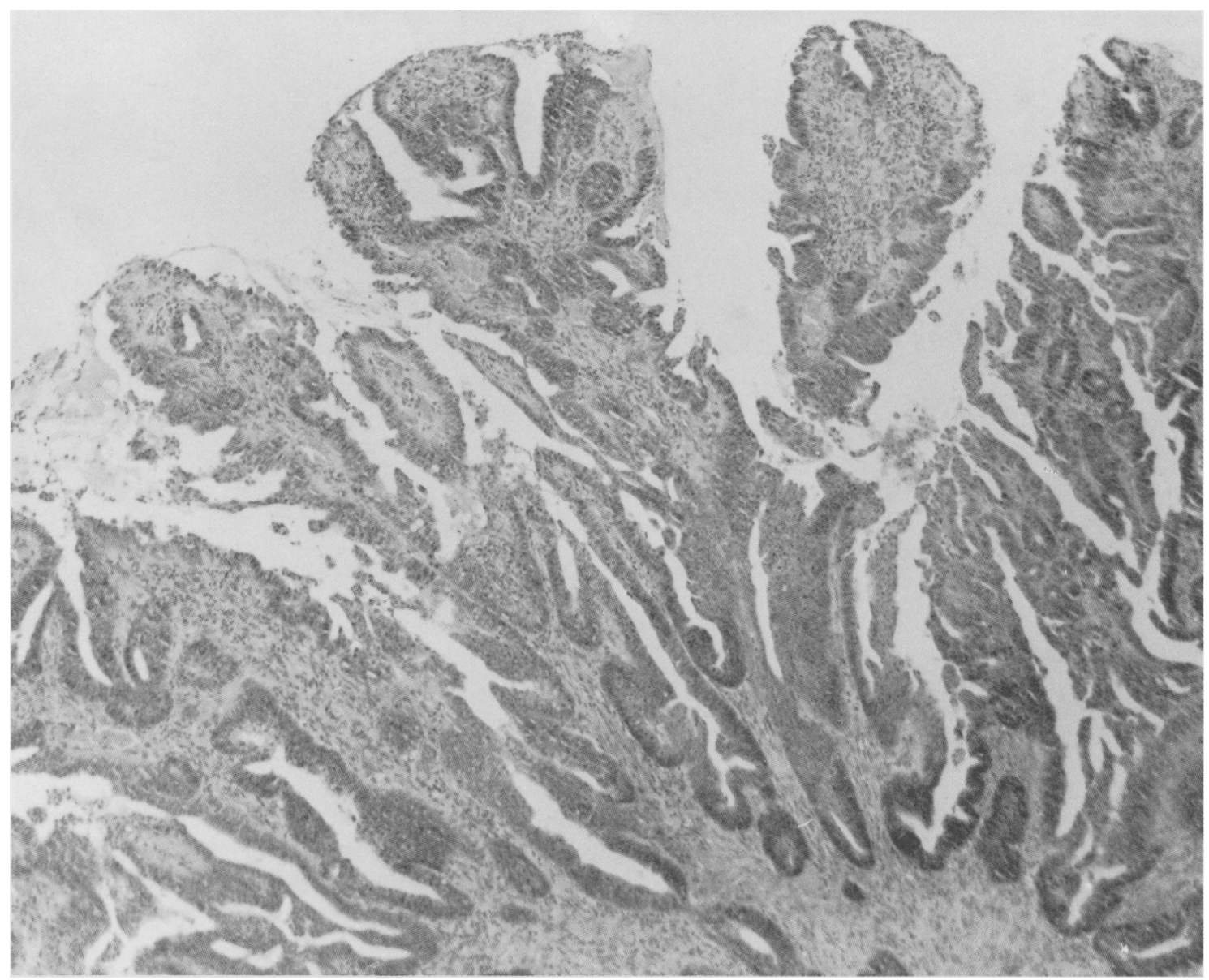

Fig. 3 Surface of the tumour demonstrating the typical appearances of a tubulovillous adenoma.

mucin production. The HID-AB stain revealed sulpho and sialo mucins in the non-neoplastic and the mucoid areas. The surface papillary areas of the tumour stained more strongly for sulpho mucins. The $\mathrm{PB} / \mathrm{KOH} / \mathrm{PAS}$ stain for o-acylated mucins was focally positive in the mucoid areas but negative in the tumour surface and non-neoplastic epithelium.

POST OPERATIVE BIOPSY

The mucosa contained sialo and sulpho acidic mucins and neutral mucin. Staining for o-acylated groups was focally positive.

The above findings suggest a focal change to large bowel type mucin within the ileostomy mucosa and within the tumour.

\section{Discussion}

Total colectomy with ileostomy may be undertaken in ulcerative colitis, Crohn's disease, familial polyposis coli, and multifocal colonic carcinoma. Ileostomy as a treatment for ulcerative colitis was first suggested by Brown of St Louis in 1913. ${ }^{4}$ The operation gained popularity in Britain in the 1940's and was combined with total colectomy. ${ }^{4} \mathrm{~A}$ report in 1970 suggested that there are at least 10000 patients with an ileostomy in Great Britain. Conventional teaching does not recognise carcinoma as a complication of a longstanding ileostomy..$^{45}$

To our knowledge there are only seven previous reports of adenocarcinoma arising at an ileostomy site. $^{6-12}$ One of these ${ }^{10}$ can be excluded from discussion on the grounds that it was not a primary carcinoma of the ileostomy and one ${ }^{4}$ arose in a villous adenoma within an ileostomy stoma after subtotal colectomy for polyposis coli with small intestinal involvement. The other five patients, like our 


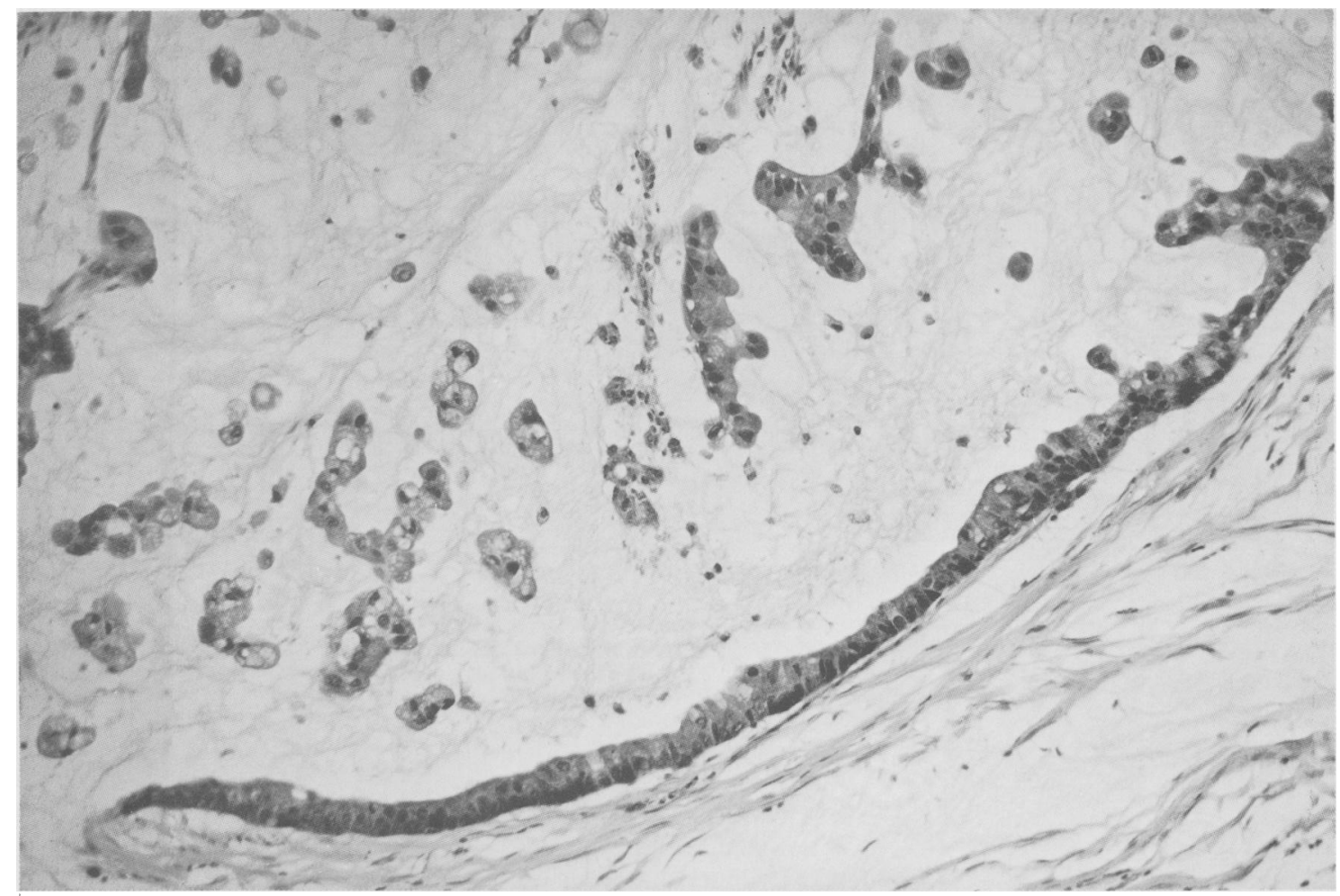

Fig. 4 (Above) Mucous cyst within the adenocarcinoma containing free floating malignant cells.

Fig. 5 (Right) Mucocutaneous junction illustrating dysplastic glandular mucosa.

patient, had ileostomies carried out for ulcerative colitis, and the carcinomas occurred from six to 31 years after the original operation. Three of these patients were described as having fungating, exophytic, or polypoid growths. ${ }^{6-8}$ This is similar to the tumour in our patient. The two remaining ${ }^{112}$ had growths that presented with induration and skin irritation and these caused considerable difficulty in diagnosis.

None of the previous reports has investigated the type of mucin within the stomal mucosa and the carcinoma. On the basis of our results we conclude that malignancy has supervened an ileal mucosa showing focal large intestinal metaplasia and severe dysplasia, which is seen at the mucocutaneous junction in Figure 5. We suggest that these mucosal changes have arisen because of longstanding irritation and chronic inflammation in the ileostomy stoma, on a similar basis to dysplasia and neoplasia occurring in the colon in cases of longstanding colitis. ${ }^{13}$ Baciewicz et al ${ }^{12}$ state that the glands in the inflamed segment of ileum in their case had undergone colonic metaplasia. Bedetti and De Risio ${ }^{6}$ state that, in their case, the ileal mucosa adjacent to the adenocarcinoma contained glands of colonic type

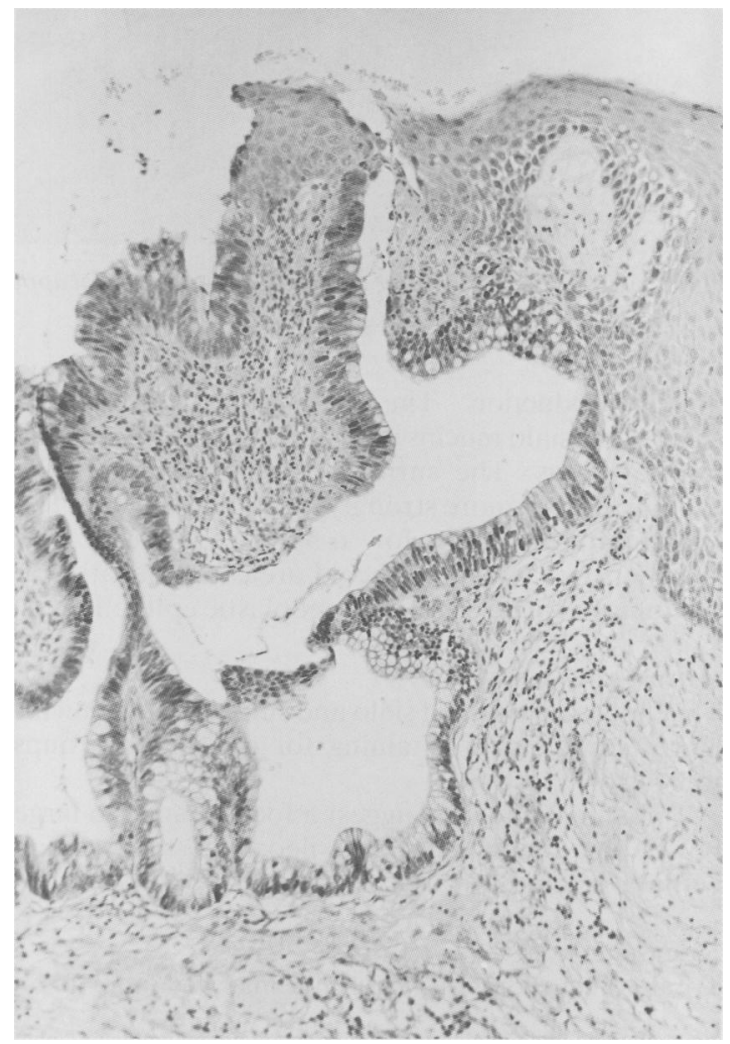




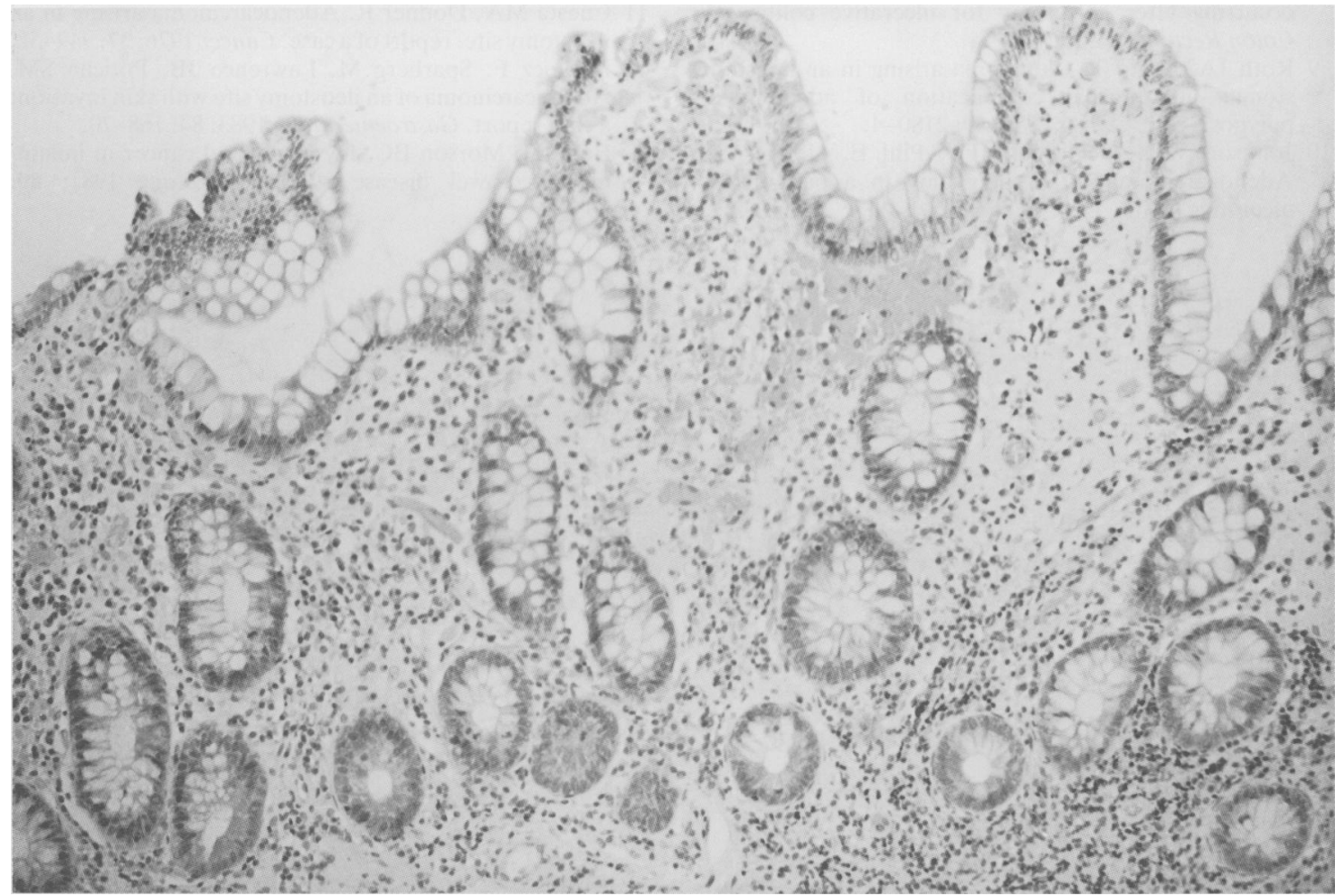

Fig. 6 Mucosal biopsy of the ileostomy following tumour resection showing loss of villous architecture, numerous goblet cells and residual Paneth cells in the crypt bases, the appearances suggesting incomplete large intestinal metaplasia. There is a mild chronic inflammatory cell infiltrate of the lamina propria.

and showed dysplastic changes, although the latter were only seen near to the tumour. Stryker ${ }^{7}$ describes moderate to severe epithelial dysplasia in ileal mucosa adjacent to a carcinoma arising after a loop ileostomy, with mild dysplasia in the distal loop, but with no dysplasia in the proximal loop.

Although rare, it must be recognised that carcinoma is a longterm complication of an ileostomy. Multiple and repeated biopsies should be done around an ileostomy of a patient who presents with fresh symptoms. Screening of asymptomatic patients with longstanding ileostomies by biopsy may be necessary for early diagnosis of mucosal dysplasia or early neoplastic change.

The authors acknowledge the help of Mr D G Ostick, Consultant Surgeon in the preparation of this paper and his permission to publish details of a patient under his care and also Mrs L Kearns for typing the manuscript.

\section{References}

1 Spicer SS. Diamine methods for differentiating mucosubstances histochemically. In Bancroft JD, Stevens A, eds. Theory and practice of histological techniques. London: Churchill Livingstone, 1982.

2 Culling CFA, Reid PE, Burton JD, Dunn WL. A histochemical method of differentiating lower gastrointestinal mucins from other mucins in primary or metastatic tumour. J Clin Pathol 1975; 28: 656-8.

3 Filipe MI. Mucins in the human gastrointestinal epithelium: a review. Invest Cell Pathol 1979; 2: 195-216.

4 Goligher JC. Surgery of the anus, rectum and colon. London: Baillière Tindall, 1981.

5 Hawley PR, Ritchie JK. The colon. Part I. Complications of ileostomy and colostomy following excisional surgery. Clinics Gastroenterol 1979; 18: 403-15.

6 Bedetti CD, De Risio VJ. Primary adenocarcinoma arising at an ileostomy site: an unusual complication after colectomy for ulcerative colitis. Dis Colon Rectum 1986; 29: 572-5.

7 Stryker SJ. Primary stomal adenocarcinoma: an unusual complication of ileostomy. Dis Colon Rectum 1983; 26: 47-9.

8 Sigler L, Jedd FL. Adenocarcinoma of the ileostomy 
occurring after colectomy for ulcerative colitis. Dis Colon Rectum 1969; 12: 45-8.

9 Roth JA, Logio T. Carcinoma arising in an ileostomy stoma: an unusual complication of adenomatous polyposis coli. Cancer 1982; 49: 2180-4.

10 Johnson WR, McDermott FT, Pihl E, Hughes ES. Adenocarcinoma of an ileostomy in a patient with ulcerative colitis. Dis Colon Rectum 1980; 23: 351-2.
11 Cuesta MA, Donner R. Adenocarcinoma arising in an ileostomy site: report of a case. Cancer 1976; 37: 949-52.

12 Bacieicz F, Sparberg M, Lawrence JB, Poticha SM. Adenocarcinoma of an ileostomy site with skin invasion: a case report. Gastroenterology 1983; 84: 168-70.

13 Butt JH, Morson BC. Dysplasia and cancer in inflammatory bowel disease. Gastroenterology 1981; 80: 865-8. 